\title{
Encystation of Giardia intestinalis-a Journey from the Duodenum to the Colon
}

\author{
Elin Einarsson $^{1} \cdot$ Staffan G. Svärd ${ }^{1}$
}

Published online: 7 July 2015

(C) Springer International Publishing AG 2015

\begin{abstract}
The intestinal protozoan parasite Giardia intestinalis has a simple life cycle consisting of diseasecausing trophozoites and infectious cysts. The parasite differentiates into the cyst stage (encystation) when duodenal trophozoites are swept further down in the small intestine. The encystation process can be divided into an early and a late phase. Expressions of cyst wall sugars and proteins are induced early during encystation, and Giardia has developed special transport systems for cyst wall material. This is combined with disassembly of the flagella and adhesive disc. In the later phase of encystation, nuclei are divided, DNA is replicated, and the parasites prepare for the next step in differentiation (excystation) via large changes in gene expression. Even if many aspects of the encystation process have been studied and much information has been gained during the last years, much still remains to be explored regarding this highly complex process.
\end{abstract}

Keywords Protozoa $\cdot$ Differentiation $\cdot$ Encystation-specific vesicle $\cdot$ Cyst $\cdot$ Cyst wall protein

\section{Introduction}

The intestinal protozoan parasite Giardia intestinalis (synonyms Giardia lamblia and Giardia duodenalis) is distributed

This article is part of the Topical Collection on Protozoa (Giardia)

Staffan G. Svärd

staffan.svard@icm.uu.se

1 Department of Cell and Molecular Biology, Uppsala University, BMC, Box 596, 75124 Uppsala, Sweden worldwide and estimated to cause 280 million symptomatic infections (giardiasis) annually [1]. The parasite is spread most often via contaminated water, and many developing countries are considered endemic regions. Symptoms of infection are variable but typically include watery diarrhea, nausea, gastric pain, and weight loss [2]. The first signs of infection appear after 6-15 days, and giardiasis is usually treated with metronidazole or other nitroimidazoles [3]. The parasite is noninvasive and secretes no known toxins, and there is currently limited understanding about the disease-causing mechanisms [2].

The parasite has a simple life cycle with two main stages, the trophozoite and the cyst, and two intermediary stages, encyzoites and excyzoites [4] (Fig. 1). The infection starts by host ingestion of infectious cysts, which are triggered to excyst by the acidic milieu in the stomach and the presence of bile and trypsin in the duodenum. The emerging excyzoites undergo cytokinesis twice, without an intervening DNA replication, giving rise to four trophozoites [5]. The binucleated trophozoites attach to the intestinal epithelium, using an adhesive disc, where they proliferate and cause disease. The parasite uses antigenic variation by expressing different variant surface-specific proteins (VSPs) to escape adaptive immunity and to prolong infection [4]. Encystation starts when the parasite is swept away further down in the small intestine, and the process can be divided into an early and late phase. The early phase involves the formation of encystation-specific vesicles (ESVs) that exclusively transport cyst wall proteins (CWPs) to the membrane for building of the cyst wall. The ESV containing cell (encyzoite) starts to change shape, and the adhesive disc together with the flagella is internalized [6]. In the late phase of encystation, the nuclei divide and undergo a round of DNA replication, giving the mature cyst four tetraploid nuclei [5]. The last step of encystation is a maturation step where the cyst wall filaments are cross-linked to generate a compact, 


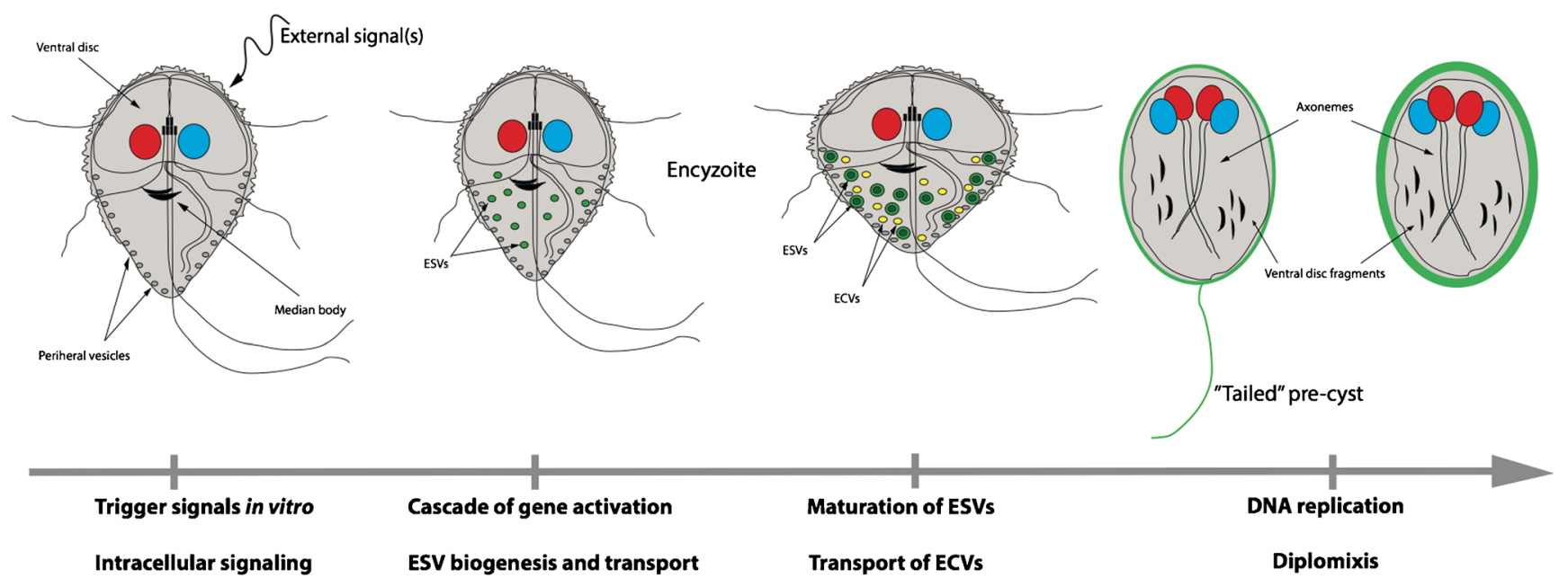

Fig. 1 Summarizing image showing the gradual transformation from the motile trophozoite via encyzoite to the final cyst stage. The trophozoite senses the external environment, and encystation is induced via not fully understood intracellular pathways. The trophozoite passes a point in early differentiation after which it is no longer possible to revert back to the proliferating stage. Transcription of encystation-specific genes is activated by different transcription factors (e.g., Myb2), among them, the three cyst wall proteins (CWP1-3). The transcription appears to be controlled on multiple levels, e.g., by epigenetic mechanisms. The production of CWPs is dramatically increased, and the transportation in encystation vesicles (ESVs) begins in early phase of the process. These vesicles undergo maturation steps after leaving the ER during which proteolytic processing of CWP2 leads to the formation of a condensed core. The

mature cell wall [6]. Cysts secreted in fecal material are effectively protected from environmental stress like UV [7] by the cyst wall and can survive several weeks in fresh water.

The encystation process is essential for transmission and survival of the parasite $G$. intestinalis. This review gives an update on recent findings regarding this fascinating cellular transformation and highlights areas where future research is needed.

\section{Induction of Encystation}

It is possible to study the entire life cycle of this parasite in vitro, and several encystation protocols have been developed for this purpose. The most used is the two-step protocol [8] where trophozoites are starved for bile prior to addition of encystation medium that contains high levels of porcine bile and lactic acid. Other protocols have also been developed which use cholesterol-depleted serum [9] or high levels of bovine bile [10], and these have been proven to be very effective in producing mature cysts. The protocols share the features of lipid starvation and an elevated $\mathrm{pH}$, which are likely stimuli of the parasite encounter when relocated to the lower part of the small intestine in the host. It is not sufficient to only increase the $\mathrm{pH}$ of the growth media to induce encystation

other component of the cyst wall, the UDP-N-acetylgalactoseamine (UDP-GalNac) sugar, is also synthesized and secreted via encystationpositive carbohydrate vesicles (ECVs). The mechanism behind assembly of the cyst wall remains unknown. Late during encystation, the cell rounds up as it approaches dormancy and the ventral disc together with the flagella is disassembled. Often, pre-cyst stages with a "tail" can be observed in encystation. Two rounds of DNA replication occur without cytokinesis rendering a cyst with four nuclei each with the genome ploidy of $4 \mathrm{~N}$. Interconnections between the nuclei in the cysts are formed, and genetic material can be exchanged through the process "diplomixis." During excystation, each cell receives one pair of nonsister nuclei (as indicated by red and blue)

[11•], so a combination of these two signals is required for encystation in vitro.

There is a very limited knowledge about how intracellular signaling is induced and mediated during encystation. Giardia possesses two homologs of extracellular regulated kinases 1 and 2 (ERK1 and ERK2), well-known MAP kinases involved in intracellular signaling in higher eukaryotes [12]. In Giardia, these proteins localize to different structures; ERK2 localizes to the nuclei and caudal flagella in trophozoites and appears mostly cytoplasmic in encysting cells, whereas ERK1 seems to be associated with the median body and the outer rim of the disc [13]. However, neither ERK1 nor ERK2 translocates to the nuclei during encystation, and so far, no transcription factors have been shown to be regulated by the giardial ERK proteins. Putative homologs of MEK and MEKK kinases are present in the genome of Giardia, but if they play a role in activating ERK1/2 remains to be investigated [13]. Even though several studies exist on individual signaling proteins [13-17], much remains to be explored to elucidate the complete signaling pathways for this parasite. Another interesting aspect is how long the encystation-inducing signals need to be present before the trophozoites commit to differentiation. A recent publication shows that trophozoites exposed to encystation medium for 3-6 h continue to encyst even if they are returned to normal growth medium [18], and hence, 
commitment is an early event in the process. Around the same time (5-8 h), ESV formation is prominent among trophozoites in the population $[11 \bullet, 19,20 \bullet \bullet$, indicating that when cyst wall production has reached a certain stage, the cells will complete the encystation process. This suggests that the parasite reaches a "point of no return" early in encystation (3-6 h) after which it is impossible to revert back to proliferation and differentiation will continue until mature cysts are generated. It also means that very early during encystation, there is a possibility for trophozoites to abort the process, if the surrounding environment becomes advantageous for growth.

The response to start encystation is heterogeneous among Giardia cells in the population in vitro; some of the cells will never induce encystation, whereas some spontaneously undergo cyst formation even in absence of encystation medium. In vivo experiments in gerbils using isolate WB show that trophozoites and encysting cells are distributed evenly in the small intestine [21]. The majority of cysts was found in the colon, but also present in last sections of the small intestine [21]. This suggests that there are a variety of cell stages present in vivo as well, where some cells do not undergo encystation and not all encysting cells complete the process. Moreover, different clones of the isolate WB undergo the encystation process differently $[10,21]$. Adding to the complexity of how encystation is triggered, it seems that assemblage A parasites proliferate and differentiate better in vitro than parasites belonging to other assemblages [4]. At the same time, these isolates grow and differentiate well in vivo [4]. Perhaps, this is due to that vital signals are missing during the in vitro conditions but present in vivo, and therefore, much could be learned regarding the signaling and what triggers the encystation process by studying parasites from different assemblages both in vitro and in vivo.

\section{Transcriptional Response During Encystation}

The promoter regions of Giardia are short and A/T-rich without any identifiable TATA-box to initiate transcription [22]. The homolog to the TATA-binding protein (TBP) found in the genome is extremely divergent, and only four of the 12 general transcription initiation factors have been reported to have giardial homologs [23]. A few giardial transcription factors have been studied in more detail, especially their role in encystation. Much focus has been put on the regulation of CWPs and enzymes involved in cyst wall biosynthesis. The first transcription factor identified and characterized is a Myb2like protein. The level of giardial Myb2 (gMyb) increases during encystation, and it localizes to the nuclei [24]. gMyb recognizes and can bind the sequence $\mathrm{C}(\mathrm{T} / \mathrm{A}) \mathrm{AC}(\mathrm{A} / \mathrm{T})(\mathrm{G} / \mathrm{C})$ which is found in the promoter regions of encystationinduced genes like $c w p 1-3$ and $g 6 p i-b$, an important enzyme in the synthesis of the $\beta(1-3)-N$-acetyl-D-galactosamine
(GalNAc) sugars in the cyst wall, as well as for gmyb2 itself [24]. Overexpression of gMyb2 leads to an increase of CWP1 on both the messenger RNA (mRNA) and protein level, and the capability to form mature cysts increases [25]. Deleting the Myb2-binding site of the promoter region of g6pi- $b$ leads to a reduction in promoter activity during encystation for this gene [24]. Moreover, when the Myb2-binding site is mutated to reduce Myb2 binding, the promoter activity is reduced during encystation. Hence, this site is important for promoter activity and the positive autoregulation of this transcription factor [25]. Interestingly, there are indications that the CWP genes might be silenced in trophozoites by a repressor mechanism allowing encystation-specific expression. Only 64 nucleotides upstream of the start codon of CWP-2 are needed for encystationspecific expression of luciferase [26]. When deleting certain elements within this region ( -64 to -23$)$, the CWP2 promoter increased its expression in trophozoites, suggesting the presence of a negative cis-acting element [26]. The authors also reported that the region contained a positive cis-acting element, as deletion of another area ( -23 to -10$)$, led to less expression in encysting cells. Several other transcription factors have been investigated and reported to play a role in the regulation of the expression of cyst wall proteins [27-33]. It remains to be investigated if and how these interact to modulate the massive induction of these genes. It would also be very interesting to knockout the transcription factors to see how that affects the differentiation process. So far, knockout experiments have been lacking in this tetraploid parasite, and it would be a vital tool to investigate several aspects of the biology of Giardia. It is important for the parasite to have a tight regulation of the encystation-specific genes since it would otherwise affect replication in the upper small intestine. Most likely, this is performed at multiple levels and further studies will show how this is accomplished, e.g., via transcription factors combined with chromatin structure and small RNAs (see below).

To date, there are two studies that describe the overall transcriptional responses during encystation. In the first study, the transcriptome of the entire life cycle was investigated using SAGE technology [34•]. Only 42 transcripts were found to be upregulated during encystation in this study: the three CWPs, several enzymes involved in UDP-GalNAc synthesis, the excyzoite surface protein high cysteine nonvariant cyst protein (HCNCp), and 13 hypothetical genes [34•]. However, two different encystation protocols were combined in this study (trophozoites to 42-h encystation via the two-step method and cysts via the high bile method). In the other study, the transcriptional response was studied during the first $7 \mathrm{~h}$ of encystation during which the CWPs are synthesized and transported from the ER in ESVs for maturation [11•]. Microarrays were used, and to avoid any protocol-induced off target effects, the authors used two different encystation protocols. The two-step method induced 29 genes whereas the cholesterol-free serum method induced 37 genes early during 
encystation, generating a core set of only 13 genes that are induced early during encystation [11•]. The core-upregulated genes all contain at least one Myb2-binding site, suggesting that the Myb-binding site is a signature motif for early phase encystation genes.

For additional information about temporal changes of gene expression during encystation and the regulation behind it, further studies are needed that include the early and later stages of the encystation process as well as mature cysts.

\section{Changes of the Proteome During Encystation}

The first overview of the Giardia proteome of encysting cells is available and gives an important complement to the existing knowledge of the transcriptional response [20••]. The study investigates the proteome during the first $14 \mathrm{~h}$ of encystation during which cells accumulate CWPs in the ER and mature ESVs are formed. The Giardia proteome was reported to be overall robust during encystation with large number of overlapping proteins between time points. The largest differences were observed in the early phases of encystation, suggesting that the regulation on the protein level is strongest at this time [20••]. Comparing samples from 0 to $12 \mathrm{~h}$ shows similar distribution of proteins with predicted cellular localization for the mitosome, nucleus, and cytosolic proteins. There was a reduction for proteins predicted to traffic to or through the ER at $4 \mathrm{~h}$, and the majority of secreted proteins found at the other time points were VSPs. An overall reduction of the VSP repertoire was found at $4 \mathrm{~h}$, consistent with the decrease of ER-targeted proteins. The data indicate that the VSP diversity is affected by encystation $[20 \bullet \cdot$. The high cysteine membrane proteins (HCMPs) are another group of predicted secreted proteins that also changed during encystation $[20 \bullet \cdot]$. Perhaps, these two groups of cysteine-rich surface proteins share a regulatory mechanism during this process. Proteins that significantly increased during encystation are involved in diverse functions such as protein folding, glycolysis, cytoskeleton regulatory components, and NEK kinases [20••]. Only modest changes were reported for the later time points $(8-12 \mathrm{~h})$ of differentiation, and it was reported that genes associated with carbohydrate biosynthesis were enriched at this time point. There were many hypothetical proteins reported in the data sets, reflecting the large number of genes present in parasite genomes with unknown function $[20 \bullet \cdot$.

Changes in the proteome involve new protein synthesis but also degradation. Proteasomes have been shown to interact with the ESV membrane early during encystation, suggesting a role for the proteasome in Giardia encystation [35]. Proteasomes with the regulatory $19 \mathrm{~S}$ particle have been purified using a tandem affinity proteomic approach [36]. This revealed that Giardia proteasomes lack the Rpn13 Ubiquitin receptor, but the other receptor, Rpn10, was found. A recent study showed localization of Rpn10 to the flagellar pores, nuclei, and cytosol in trophozoites. However, the association to the flagellar pores was not evident in encysting cells [37].

Ubiquitination and, in particular, the ubiquitin-activating enzyme E1 seem to be involved in the differentiation process. The expression of E1 is regulated on both transcriptional and protein level during encystation, and overexpression of the protein yielded increased encystation rate. The localization of the enzyme appears to be associated to the cyst wall and as cytoplasmic spots in mature cysts [38]. The SUMO modification is similar to ubiquitin, but not used for tagging proteins for degradation by the proteasome. SUMOylation of the metabolic enzyme arginine deiminase (ADI) improves translocation from the cytoplasm to the nuclei during late encystation. Inside the nuclei, ADI functions as a peptidyl arginine deiminase (PAD) and is suggested to downregulate the transcription of CWP2 [39]. The activity of acyltransferases and the reversible modification S-palmitoylation during encystation have been studied [40]. Several potential acyltransferases were found in the genome, and they are expressed in trophozoites as well as encysting parasites. During encystation, the levels of expression of CWPs and ESV formation differed among parasite lines overexpressing different acyltransferases, but proteomic analysis would be needed to find the targets of these acyltransferases and how these contribute during encystation [40].

To date, there are no proteomic studies that include the complete transformation from trophozoite to mature cyst. It will be important to expand the knowledge about changes in the proteome and connect it to the identified transcriptional changes during Giardia encystation. Large-scale analyses of post-translation modifications can complement this and generate a complete picture of encystation-induced gene expression changes.

\section{Building of the Cyst Wall}

The most striking change in secretion of proteins occurs during the early phase of encystation when massive amounts of cyst wall components are transported from the ER to the plasma membrane. The cyst wall material is transported in ESVs, and the formation and transport of these have been extensively studied [41, 42] since they were discovered [43]. The waterresistant insoluble cyst wall is made of $36 \%$ protein and $63 \%$ GalNAc homopolymer [44]. The cyst wall protein family consists of three members, which share several features $[45,46]$. The presence of an N-terminal signal peptide targets them to the secretory pathway, and all three contain a central leucinerich repeat (LRR) region important for sorting into the ESVs [45]. CWP1 and 2 carry five tandem LRRs whereas CWP3 has four complete and one incomplete repeat regions [46]. A cysteine-rich domain is found in the $\mathrm{C}$-terminal region of the 
CWPs, and CWP2 is different compared to the other two by the presence of a basic extension in this region [45]. CWPs peak in expression on the mRNA level at approximately 7-h post-induction, but an accumulation of newly synthesized protein appears in the ER after about 2-h post-induction. The cargo of ESVs is exclusively cyst wall material and no other secreted proteins, strongly suggest sorting in the ER prior to export [42]. Giardia lacks a constitutively expressed Golgi apparatus, and the features of ESVs indicate that these organelles act as a stage-induced cis Golgi. The trafficking process of cyst wall material, from the ER to the ESV, appears to be dependent on COPII coat formation and the small GTPase Sar1 [35]. Recently, it was shown that ER exit sites (ERES) induce ESV biogenesis since CWP1 depends on functional ERES for accurate trafficking [47••]. Furthermore, the colocalization of the nascent ESVs and ERES suggests a role of ERES in ESV biogenesis [47••]. The CWPs are not directly secreted after completion of ESV formation but, instead, delayed for several hours and converted into a trans-Golgi-like tubular vesicular network during late encystation [35]. This is presumably to allow post-translational modifications prior to secretion at the cell surface. A number of modifications have been reported such as formation of disulfide bonds, isopeptide-linkages, and addition of phospho-groups [48-50]. Another modification is the proteolytic processing of the basic extension of CWP2. Dual-tagged CWP2 and CWP/GFP chimeras have been used to study processing of the proteinaceous portion of the cyst wall material (CVM) in ESVs [51••]. The cell wall material $(\mathrm{CWM})$ is partitioned into two distinct phases; CWP3 forms a condensed core together with the processed form of CWP2, and the mature N-terminal CWP2 together with CWP1 remains in fluid state until secretion. The fluid part is secreted rapidly whereas the condensed part of the CWM is secreted slowly for hours [51••]. This indicates that the formation of the cyst wall is a highly complex process.

A part from the CWPs, a few other proteins have been found to localize to the ESVs. Among them is an HCNCp that localized to ESVs and secreted to the plasma membrane-cyst wall interface and stayed on the surface of the excyzoite in the cyst [52]. Another group of cysteine-rich proteins (tenascinlike proteins) was also studied, and they appear to mainly localize not only to the cyst wall but also to the excyzoite surface [53].

The lysosomal-like peripheral vesicles (PVs) and ESVs were subjected to flow cytometry organelle sorting coupled to mass spectrometry aiming to find additional proteins associated to these organelles [54••]. The proteome data set from the ESV-enriched fraction revealed a candidate set of 72 proteins. Among these, many had ER localizations and the membranes of the two-organelle systems appear closely connected. Existing evidence of connections between ESVs allowing exchange of CWM makes it more challenging to define ESV boundaries [35]. Several ribosomal proteins were enriched in the ESV fraction, and ribosomes were recruited to ESV membranes during differentiation. A possible explanation could be the presence of co-translational insertion of proteins from the ER across ESV membranes, but further experiments are needed for validation [54••].

While the CWPs are transported from the ER to the surface by ESVs, much less is known about the transport and incorporation of the UDP-GalNAc sugar in the cyst wall. This Giardia-unique carbohydrate is synthesized de novo by a pathway containing five enzymes of bacterial origin $[55,56]$ using endogenous glucose as starting molecule. The enzymes are transcriptionally and allosterically upregulated during encystation [55]. During the final step of synthesis, the UDP-GalNAc is polymerized by an activity named the "cyst wall synthetase" [57]. Even if evidence exists for the presence of this enzyme via activity measurements, it still remains unknown which gene(s) that encode this protein. The structure and assembly of the cyst wall have been under investigation, and the homopolymer of GalNAc appears as curled fibrils compressed by CWPs [58]. Both CWP1 and CWP2 can bind the fibrils, and the LRR of CWP1 is reported to have lectinbinding properties [58]. In the same study, the GalNAc homopolymer was visualized using the binding properties of recombinant CWP1 and reported to be present in small vesicles of encysting Giardia. These vesicles did not co-localize with ESVs, suggesting a different transportation pathway of the sugar components of the cyst wall [58]. In line with this result, a recent study proposes the presence of carbohydrate-positive vesicles (ECVs) in encysting parasites [59]. The authors found electron-translucent vesicles that stain positive for carbohydrate content, and binding of the lectin DBA indicated the presence of GalNAc [59]. Localization studies of putative the UDP-GlcNAc 4'-epimerase and a nucleotide sugar transporter were performed based on their presence in ESV proteomics data [54••]. The epimerase is associated to the ER and the transporter mainly to perinuclear ER and early ESVs pointing toward synthesis of UDP-GalNAc in the ER [54••].

Further studies are needed to clarify how the ESVs and ECVs are connected and to identify the mechanism behind cyst wall assembly.

\section{Changes in Lipid Metabolism During Differentiation}

The large changes in vesicle transport during encystation require production of new membrane structures. Being a parasite, Giardia has a limited range of metabolic pathways [56] and depends on uptake of lipids and fatty acids from the environment [60]. The sphingolipid ceramide cannot be synthesized de novo and is taken up by the parasite using clathrinmediated pathways [61]. Only three synthesis-related genes 
and two metabolic genes from the sphingolipid pathway are present in Giardia [62]. These genes are differentially expressed, and the glycosylceramide transferase (GlcT1) is only expressed in encysting parasites [62]. By modulating the levels of GlcT1, using a specific inhibitor, the levels of encystation and cyst production were reduced. This shows the importance of glucosylceramide synthesis during the process of encystation $[62,63]$. Further studies using morpholino knockdown of the enzyme showed abolished ESV formation and generated nonviable cysts [64]. Most recently, the presence of cholesterol and GM ganglioside-enriched raft-like domains was shown in the plasma membrane of Giardia trophozoites [65.]. Lipid rafts are found in the plasma membrane of many eukaryotes and are involved in various processes e.g., cell adhesion, membrane signaling, and differentiation [66]. The cholesterol-binding drugs nystatin and filipin III disrupt the lipid rafts in Giardia trophozoites, interfere with ESV formation, and alter the morphology of cysts [65•]. It was suggested that the raft-like domains require cholesterol to maintain integrity, which appears critical for encystation [65•]. The exact function and potential involvement in signaling of the lipid rafts during encystation remain unknown.

\section{Fusion of Nuclei Occurs During Late Encystation}

The binucleated trophozoite alternates from a diploid $(2 \times 2 \mathrm{~N})$ to a tetraploid $(2 \times 4 \mathrm{~N})$ genome content during the cell cycle. The G2 phase is the longest phase of the cell cycle, and it contains the restriction point to start differentiating into the cyst form $[5,67]$. During encystation, the two nuclei divide without cytokinesis forming a pre-cyst with ploidy of $4 \times 2 \mathrm{~N}$ followed by another round of replication to the final ploidy of $16 \mathrm{~N}(4 \mathrm{x} 4 \mathrm{~N})$ in the mature cyst [5]. During excystation, the excyzoite goes through cellular division twice without DNA replication, in the end, generating four trophozoites [5]. Giardia is considered to be asexual, but several homologs to genes involved with meiosis in other eukaryotes are found in the genome [4]. Moreover, the genome of WB isolate contains low levels of allelic sequence heterozygosity (ASH) which is surprising for an asexual organism that lack control of the buildup of differences between nuclei $[56,68]$. This suggested that the nuclei can be connected and put the cyst nuclei into focus. Evidence for fusion of the nuclei and exchange of genetic material in the form of episomal plasmids was discovered during encystation [69]. The authors named this event "diplomixis," and three meiosis genes were reported to be expressed late in encystation to facilitate homologous recombination [69]. Later, it was shown that chromosomally integrated markers were exchanged between nuclei during differentiation [70]. By using FISH to follow the integrated markers, it was shown that daughter trophozoites inherit one copy of each parental nucleus after excystation [70]. The recombination events occurring during encystation by diplomixis could be involved in reducing ASH between nuclei [70].

The mechanisms behind the nuclear division were further investigated by [71]. Nuclear division occurs by semi-open mitosis, resulting in four daughter nuclei from two nonsister pairs. The pairs observed are interconnected with nuclear membrane bridges that remain in the excyzoites [71]. After division of excyzoite, each trophozoite inherits one pair of nonsister nuclei, in line with the model proposed by [70].

\section{Regulation of Differentiation Is Likely to Occur at Multiple Levels}

The fact that very few transcription factors have been found for this parasite (see above) might be an indication that the regulation of gene expression occurs at multiple levels. The process of encystation is complex and most likely entails sequential activation of genes required for successful development into the cyst stage. The details of this regulation remain incomplete.

Epigenetic changes are involved in the encystation process, and histone acetylation decreased during encystation [72]. The histone acetylation levels were modified using a histone deacetylase (HDAC) inhibitor resulting in increased levels of histone acetylation, alteration of gene expression, and blocked cyst formation [72]. The results clearly indicate that chromatin changes are part of dictating the transcriptional response of this parasite differentiation process. Giardia possesses several SF2 RNA helicases [73], and in higher eukaryotes, RNA helicases can interact with HDACs [74]. The expression level of two DEAD-box helicases increased greatly during encystation conditions. Authors also predict that helicases can be involved in the RNAi pathway present in Giardia [75] and participate in post-transcriptional silencing of genes [73].

Antigenic variation appears to be regulated by RNAirelated pathway and/or microRNAs [75, 76]. Recently, it was investigated if small RNAs can be involved in regulation of encystation [77•]. Two time points during encystation as well as trophozoites and cysts were analyzed by deep sequencing. Increasing levels of endogenous small interfering RNA (siRNA) were reported during the process originating from telomeric retrotransposons and from specific clusters in the genome [77•]. A knockdown strain of giardial Dicer was used in encystation and reported to be impaired in cyst formation, indicating a role of siRNAs in the process since Dicer is required for siRNA biogenesis. However, none of the targets predicted for the siRNAs has been experimentally verified. The siRNA data should be compared to transcriptional response of mRNA and protein expression to get a complete picture of their function during encystation. In addition, tRNA-derived siRNA was also found to increase during late 
encystation, but their function during differentiation is difficult to speculate about without further studies [77•].

\section{Conclusions}

The complete life cycle of the parasite $G$. intestinalis can be studied in vitro $[9,10,43]$ and has generated the possibility to study many vital aspects of encystation. However, the exact trigger and pathways responsible for induction of the process remain unknown. There are some indications of important players in the signaling pathway, but further studies using specific inhibitors are needed to investigate the exact role. Another possibility is to study the process in other assemblages, such as the GS isolate, or possibly even during in vivo conditions.

Many aspects of early encystation have been studied, including the biogenesis and transport of ESVs [41, 42]. It will be interesting to investigate the detail of how the carbohydrates are transported in ECVs [59] and the interactions between CWPs that must occur to build the cyst wall. Perhaps, the finding of the gene encoding the cyst wall synthetase will help to gain information regarding this process.

Transcriptional profiles coupled with proteomics ranging over the entire encystation process, including mature cysts, would improve current knowledge of temporal activation of genes and their protein product. Future studies are also needed to elucidate how genes are regulated during encystation, which appear to be orchestrated on multiple levels [72, 77•].

Lastly, the possibility to do knockout experiments on specific genes will improve the knowledge about the role certain proteins play during the journey in the small intestine from trophozoite to a cyst.

\section{Compliance with Ethics Guidelines}

Conflict of Interest Elin Einarsson and Staffan G. Svärd declare that they have no conflict of interest.

Human and Animal Rights and Informed Consent This article does not contain any studies with human or animal subjects performed by any of the authors.

\section{References}

Papers of particular interest, published recently, have been highlighted as:

- Of importance

•- Of major importance

1. Lane S, Lloyd D. Current trends in research into the waterborne parasite Giardia. Crit Rev Microbiol. 2002;28:123-47. doi:10. 1080/1040-840291046713.
2. Halliez MCM, Buret AG. Extra-intestinal and long term consequences of Giardia duodenalis infections. World J Gastroenterol WJG. 2013;19:8974-85. doi:10.3748/wjg.v19.i47.8974.

3. Escobedo AA, Hanevik K, Almirall P, Cimerman S, Alfonso M. Management of chronic Giardia infection. Expert Rev Anti Infect Ther. 2014;12:1143-57. doi:10.1586/14787210.2014.942283.

4. Ankarklev J, Jerlström-Hultqvist J, Ringqvist E, Troell K, Svärd SG. Behind the smile: cell biology and disease mechanisms of Giardia species. Nat Rev Microbiol. 2010;8:413-22. doi:10.1038/ nrmicro2317.

5. Bernander R, Palm JE, Svärd SG. Genome ploidy in different stages of the Giardia lamblia life cycle. Cell Microbiol. 2001;3: $55-62$.

6. Erlandsen SL, Macechko PT, van Keulen H, Jarroll EL. Formation of the Giardia cyst wall: studies on extracellular assembly using immunogold labeling and high resolution field emission SEM. J Eukaryot Microbiol. 1996;43:416-29.

7. Einarsson E, Svärd SG, Troell K. UV irradiation responses in Giardia intestinalis. Exp Parasitol. 2015;154:25-32. doi:10.1016/j. exppara.2015.03.024.

8. Boucher SE, Gillin FD. Excystation of in vitro-derived Giardia lamblia cysts. Infect Immun. 1990;58:3516-22.

9. Luján HD, Mowatt MR, Byrd LG, Nash TE. Cholesterol starvation induces differentiation of the intestinal parasite Giardia lamblia. Proc Natl Acad Sci U S A. 1996;93:7628-33.

10. Kane AV, Ward HD, Keusch GT, Pereira ME. In vitro encystation of Giardia lamblia: large-scale production of in vitro cysts and strain and clone differences in encystation efficiency. J Parasitol. 1991;77: 974-81.

11. Morf L, Spycher C, Rehrauer H, Fournier CA, Morrison HG, Hehl $\mathrm{AB}$. The transcriptional response to encystation stimuli in Giardia lamblia is restricted to a small set of genes. Eukaryot Cell. 2010;9: 1566-76. doi:10.1128/EC.00100-10. First microarray study of the transcriptional changes during the encystation process. identified a set of conserved early upregulated genes and the importance of the Myb transcription factor.

12. English J, Pearson G, Wilsbacher J, Swantek J, Karandikar M, Xu S, et al. New insights into the control of MAP kinase pathways. Exp Cell Res. 1999;253:255-70. doi:10.1006/excr.1999.4687.

13. Ellis JG, Davila M, Chakrabarti R. Potential involvement of extracellular signal-regulated kinase 1 and 2 in encystation of a primitive eukaryote, Giardia lamblia. Stage-specific activation and intracellular localization. J Biol Chem. 2003;278:1936-45. doi:10.1074/jbc. M209274200.

14. Gibson C, Schanen B, Chakrabarti D, Chakrabarti R. Functional characterisation of the regulatory subunit of cyclic AMP-dependent protein kinase a homologue of Giardia lamblia: differential expression of the regulatory and catalytic subunits during encystation. Int J Parasitol. 2006;36:7919. doi:10.1016/j.ijpara.2005.11.008.

15. Bazán-Tejeda ML, Argüello-García R, Bermúdez-Cruz RM, Robles-Flores M, Ortega-Pierres G. Protein kinase C isoforms from Giardia duodenalis: identification and functional characterization of a beta-like molecule during encystment. Arch Microbiol. 2007;187:55-66. doi:10.1007/s00203-006-0174-9.

16. Kim K-T, Mok MTS, Edwards MR. Protein kinase B from Giardia intestinalis. Biochem Biophys Res Commun. 2005;334:333-41. doi:10.1016/j.bbrc.2005.06.106.

17. Lauwaet T, Davids BJ, Torres-Escobar A, Birkeland SR, Cipriano MJ, Preheim SP, et al. Protein phosphatase 2A plays a crucial role in Giardia lamblia differentiation. Mol Biochem Parasitol. 2007;152: 80-9. doi:10.1016/j.molbiopara.2006.12.001.

18. Sulemana A, Paget TA, Jarroll EL. Commitment to cyst formation in Giardia. Microbiol Read Engl. 2014;160:330-9. doi:10.1099/ mic.0.072405-0. 
19. Hehl AB, Marti M, Köhler P. Stage-specific expression and targeting of cyst wall protein-green fluorescent protein chimeras in Giardia. Mol Biol Cell. 2000;11:1789-800.

20.• Faso C, Bischof S, Hehl AB. The proteome landscape of Giardia lamblia encystation. PLoS One. 2013;8:e83207. doi:10.1371/ journal.pone.0083207. First large-scale proteomic analysis of the encystation process. Identifies a large number of proteins with known and putative roles in encystation. This will be the start of a large number of follow-up studies of proteins with changed expression during encystation.

21. Campbell JD, Faubert GM. Comparative studies on Giardia lamblia encystation in vitro and in vivo. J Parasitol. 1994;80:36-44.

22. Adam RD. Biology of Giardia lamblia. Clin Microbiol Rev. 2001;14:447-75. doi:10.1128/CMR.14.3.447-475.2001.

23. Best AA, Morrison HG, McArthur AG, Sogin ML, Olsen GJ. Evolution of eukaryotic transcription: insights from the genome of Giardia lamblia. Genome Res. 2004;14:1537-47. doi:10.1101/ gr.2256604.

24. Sun C-H, Palm D, McArthur AG, Svärd SG, Gillin FD. A novel Myb-related protein involved in transcriptional activation of encystation genes in Giardia lamblia. Mol Microbiol. 2002;46: 971-84.

25. Huang Y-C, Su L-H, Lee GA, Chiu P-W, Cho C-C, Wu J-Y, et al. Regulation of cyst wall protein promoters by Myb2 in Giardia lamblia. J Biol Chem. 2008;283:31021-9. doi:10.1074/jbc. M805023200.

26. Davis-Hayman SR, Hayman JR, Nash TE. Encystation-specific regulation of the cyst wall protein 2 gene in Giardia lamblia by multiple cis-acting elements. Int J Parasitol. 2003;33:1005-12.

27. Wang C-H, Su L-H, Sun C-H. A novel ARID/Bright-like protein involved in transcriptional activation of cyst wall protein 1 gene in Giardia lamblia. J Biol Chem. 2007;282:8905-14. doi:10.1074/jbc. M611170200.

28. Sun C-H, Su L-H, Gillin FD. Novel plant-GARP-like transcription factors in Giardia lamblia. Mol Biochem Parasitol. 2006;146:4557. doi:10.1016/j.molbiopara.2005.10.017.

29. Su L-H, Pan Y-J, Huang Y-C, Cho C-C, Chen C-W, Huang S-W, et al. A novel E2F-like protein involved in transcriptional activation of cyst wall protein genes in Giardia lamblia. J Biol Chem. 2011;286:34101-20. doi:10.1074/jbc.M111.280206.

30. Chuang S-F, Su L-H, Cho C-C, Pan Y-J, Sun C-H. Functional redundancy of two Pax-like proteins in transcriptional activation of cyst wall protein genes in Giardia lamblia. PLoS One. 2012;7: e30614. doi:10.1371/journal.pone.0030614.

31. Lin B-C, Su L-H, Weng S-C, Pan Y-J, Chan N-L, Li T-K, et al. DNA topoisomerase II is involved in regulation of cyst wall protein genes and differentiation in Giardia lamblia. PLoS Negl Trop Dis. 2013; 7:e2218. doi:10.1371/journal.pntd.0002218.

32. Pan Y-J, Cho C-C, Kao Y-Y, Sun C-H. A novel WRKY-like protein involved in transcriptional activation of cyst wall protein genes in Giardia lamblia. J Biol Chem. 2009;284:17975-88. doi:10.1074/ jbc.M109.012047.

33. Worgall TS, Davis-Hayman SR, Magana MM, Oelkers PM, Zapata F, Juliano RA, et al. Sterol and fatty acid regulatory pathways in a Giardia lamblia-derived promoter: evidence for SREBP as an ancient transcription factor. J Lipid Res. 2004;45:981-8. doi:10.1194/ jlr.M400024-JLR200.

34. Birkeland SR, Preheim SP, Davids BJ, Cipriano MJ, Palm D, Reiner DS, et al. Transcriptome analyses of the Giardia lamblia life cycle. Mol Biochem Parasitol. 2010;174:62-5. doi:10.1016/j. molbiopara.2010.05.010. First study of transcriptional changes during the whole Giardia life cycle. Shows that the parasite prepares for excystation in the end of encystation.

35. Stefanic S, Morf L, Kulangara C, Regös A, Sonda S, Schraner E, et al. Neogenesis and maturation of transient Golgi-like cisternae in a simple eukaryote. J Cell Sci. 2009;122:2846-56. doi:10.1242/jcs. 049411.

36. Jerlström-Hultqvist J, Stadelmann B, Birkestedt S, Hellman U, Svärd SG. Plasmid vectors for proteomic analyses in Giardia: purification of virulence factors and analysis of the proteasome. Eukaryot Cell. 2012;11:864-73. doi:10.1128/EC.00092-12.

37. Sinha A, Datta SP, Ray A, Sarkar S. A reduced VWA domaincontaining proteasomal ubiquitin receptor of Giardia lamblia localizes to the flagellar pore regions in microtubule-dependent manner. Parasit Vectors. 2015;8:120. doi:10.1186/s13071-015-0737-1.

38. Niño CA, Prucca CG, Chaparro J, Luján HD, Wasserman M. The ubiquitin-activating enzyme (E1) of the early-branching eukaryote Giardia intestinalis shows unusual proteolytic modifications and play important roles during encystation. Acta Trop. 2012;123:39 46. doi:10.1016/j.actatropica.2012.03.012.

39. Vranych CV, Rivero MR, Merino MC, Mayol GF, Zamponi N, Maletto BA, et al. SUMOylation and deimination of proteins: two epigenetic modifications involved in Giardia encystation. Biochim Biophys Acta. 1843;2014:1805-17. doi:10.1016/j.bbamcr.2014. 04.014 .

40. Merino MC, Zamponi N, Vranych CV, Touz MC, Rópolo AS. Identification of Giardia lamblia DHHC proteins and the role of protein S-palmitoylation in the encystation process. PLoS Negl Trop Dis. 2014;8:e2997. doi:10.1371/journal.pntd.0002997.

41. Lujan HD. Mechanisms of adaptation in the intestinal parasite Giardia lamblia. Essays Biochem. 2011;51:177-91. doi:10.1042/ bse0510177.

42. Faso $\mathrm{C}, \mathrm{Hehl} \mathrm{AB}$. Membrane trafficking and organelle biogenesis in Giardia lamblia: use it or lose it. Int J Parasitol. 2011;41:471-80. doi:10.1016/j.ijpara.2010.12.014

43. Gillin FD, Reiner DS, Gault MJ, Douglas H, Das S, Wunderlich A, et al. Encystation and expression of cyst antigens by Giardia lamblia in vitro. Science. 1987;235:1040-3.

44. Gerwig GJ, van Kuik JA, Leeflang BR, Kamerling JP, Vliegenthart JFG, Karr CD, et al. The Giardia intestinalis filamentous cyst wall contains a novel beta(1-3)-N-acetyl-D-galactosamine polymer: a structural and conformational study. Glycobiology. 2002;12:499505.

45. Luján HD, Mowatt MR, Conrad JT, Bowers B, Nash TE. Identification of a novel Giardia lamblia cyst wall protein with leucine-rich repeats. Implications for secretory granule formation and protein assembly into the cyst wall. J Biol Chem. 1995;270: 29307-13.

46. Sun C-H, McCaffery JM, Reiner DS, Gillin FD. Mining the Giardia lamblia genome for new cyst wall proteins. J Biol Chem. 2003;278: 21701-8. doi:10.1074/jbc.M302023200.

47.• Faso C, Konrad C, Schraner EM, Hehl AB. Export of cyst wall material and Golgi organelle neogenesis in Giardia lamblia depend on endoplasmic reticulum exit sites. Cell Microbiol. 2013;15:53753. doi:10.1111/cmi.12054. Paper showing the importance of ER exit sites in the formation of encystation-specific vesicles (ESVs).

48. Reiner DS, McCaffery JM, Gillin FD. Reversible interruption of Giardia lamblia cyst wall protein transport in a novel regulated secretory pathway. Cell Microbiol. 2001;3:459-72.

49. Slavin I, Saura A, Carranza PG, Touz MC, Nores MJ, Luján HD. Dephosphorylation of cyst wall proteins by a secreted lysosomal acid phosphatase is essential for excystation of Giardia lamblia. Mol Biochem Parasitol. 2002;122:95-8.

50. Davids BJ, Mehta K, Fesus L, McCaffery JM, Gillin FD. Dependence of Giardia lamblia encystation on novel transglutaminase activity. Mol Biochem Parasitol. 2004;136:173-80.

$51 . \bullet$ Konrad C, Spycher C, Hehl AB. Selective condensation drives partitioning and sequential secretion of cyst wall proteins in differentiating Giardia lamblia. PLoS Pathog. 2010;6:e1000835. doi:10. 1371/journal.ppat.1000835. Paper showing that the ESV cargo is 
processed and separated into different phases during the transport to the plasma membrane. Shows the complex, induced protein transport during encystation.

52. Davids BJ, Reiner DS, Birkeland SR, Preheim SP, Cipriano MJ, McArthur AG, et al. A new family of giardial cysteine-rich nonVSP protein genes and a novel cyst protein. PLoS One. 2006;1:e44. doi:10.1371/journal.pone.0000044.

53. Chiu P-W, Huang Y-C, Pan Y-J, Wang C-H, Sun C-H. A novel family of cyst proteins with epidermal growth factor repeats in Giardia lamblia. PLoS Negl Trop Dis. 2010;4:e677. doi:10.1371/ journal.pntd.0000677.

54.• Wampfler PB, Tosevski V, Nanni P, Spycher C, Hehl AB. Proteomics of secretory and endocytic organelles in Giardia lamblia. PLoS One. 2014;9:e94089. doi:10.1371/journal.pone. 0094089. First proteomic analysis of ESVs. Several new putative ESV cargo proteins identified.

55. Lopez AB, Sener K, Jarroll EL, van Keulen H. Transcription regulation is demonstrated for five key enzymes in Giardia intestinalis cyst wall polysaccharide biosynthesis. Mol Biochem Parasitol. 2003;128:51-7.

56. Morrison HG, McArthur AG, Gillin FD, Aley SB, Adam RD, Olsen GJ, et al. Genomic minimalism in the early diverging intestinal parasite Giardia lamblia. Science. 2007;317:1921-6. doi:10. 1126/science.1143837.

57. Karr CD, Jarroll EL. Cyst wall synthase: Nacetylgalactosaminyltransferase activity is induced to form the novel $\mathrm{N}$-acetylgalactosamine polysaccharide in the Giardia cyst wall. Microbiol Read Engl. 2004;150:1237-43.

58. Chatterjee A, Carpentieri A, Ratner DM, Bullitt E, Costello CE, Robbins PW, et al. Giardia cyst wall protein 1 is a lectin that binds to curled fibrils of the GalNAc homopolymer. PLoS Pathog. 2010;6:e1001059. doi:10.1371/journal.ppat.1001059.

59. Midlej V, Meinig I, de Souza W, Benchimol M. A new set of carbohydrate-positive vesicles in encysting Giardia lamblia. Protist. 2013;164:261-71. doi:10.1016/j.protis.2012.11.001.

60. Mohareb EW, Rogers EJ, Weiner EJ, Bruce JI. Giardia lamblia: phospholipid analysis of human isolates. Ann Trop Med Parasitol. 1991;85:591-7.

61. Hernandez Y, Castillo C, Roychowdhury S, Hehl A, Aley SB, Das $\mathrm{S}$. Clathrin-dependent pathways and the cytoskeleton network are involved in ceramide endocytosis by a parasitic protozoan, Giardia lamblia. Int J Parasitol. 2007;37:21-32. doi:10.1016/j.ijpara.2006. 09.008 .

62. Hernandez Y, Shpak M, Duarte TT, Mendez TL, Maldonado RA, Roychowdhury S, et al. Novel role of sphingolipid synthesis genes in regulating giardial encystation. Infect Immun. 2008;76:2939-49. doi:10.1128/IAI.00116-08.

63. Sonda S, Stefanic S, Hehl AB. A sphingolipid inhibitor induces a cytokinesis arrest and blocks stage differentiation in Giardia lamblia. Antimicrob Agents Chemother. 2008;52:563-9. doi:10. 1128/AAC.01105-07.

64. Mendez TL, De Chatterjee A, Duarte TT, Gazos-Lopes F, RoblesMartinez L, Roy D, et al. Glucosylceramide transferase activity is critical for encystation and viable cyst production by an intestinal protozoan, Giardia lamblia. J Biol Chem. 2013;288:16747-60. doi: 10.1074/jbc.M112.438416.
65. De Chatterjee A, Mendez TL, Roychowdhury S, Das S. The assembly of GM1 glycolipid- and cholesterol-enriched raft-like membrane microdomains is important for giardial encystation. Infect Immun. 2015;83:2030-42. doi:10.1128/IAI.03118-14. First study showing the presence and importance of raft-like microdomains in the Giardia plasma membrane. Drugs that interfere with formation of microdomains block formation of ESVs.

66. Head BP, Patel HH, Insel PA. Interaction of membrane/lipid rafts with the cytoskeleton: impact on signaling and function: membrane/lipid rafts, mediators of cytoskeletal arrangement and cell signaling. Biochim Biophys Acta. 1838;2014:532-45. doi:10. 1016/j.bbamem.2013.07.018

67. Reiner DS, Ankarklev J, Troell K, Palm D, Bernander R, Gillin FD, et al. Synchronisation of Giardia lamblia: identification of cell cycle stage-specific genes and a differentiation restriction point. Int $\mathrm{J}$ Parasitol. 2008;38:935-44. doi:10.1016/j.ijpara.2007.12.005.

68. Jerlström-Hultqvist J, Franzén O, Ankarklev J, Xu F, Nohýnková E, Andersson JO, et al. Genome analysis and comparative genomics of a Giardia intestinalis assemblage E isolate. BMC Genomics. 2010;11:543. doi:10.1186/1471-2164-11-543.

69. Poxleitner MK, Carpenter ML, Mancuso JJ, Wang C-JR, Dawson SC, Cande WZ. Evidence for karyogamy and exchange of genetic material in the binucleate intestinal parasite Giardia intestinalis. Science. 2008;319:1530-3. doi:10.1126/science.1153752.

70. Carpenter ML, Assaf ZJ, Gourguechon S, Cande WZ. Nuclear inheritance and genetic exchange without meiosis in the binucleate parasite Giardia intestinalis. J Cell Sci. 2012;125:2523-32. doi:10. 1242/jcs. 103879.

71. Jiráková K, Kulda J, Nohýnková E. How nuclei of Giardia pass through cell differentiation: semi-open mitosis followed by nuclear interconnection. Protist. 2012;163:465-79. doi:10.1016/j.protis. 2011.11.008.

72. Sonda S, Morf L, Bottova I, Baetschmann H, Rehrauer H, Caflisch A, et al. Epigenetic mechanisms regulate stage differentiation in the minimized protozoan Giardia lamblia. Mol Microbiol. 2010;76:48 67. doi:10.1111/j.1365-2958.2010.07062.x.

73. Gargantini PR, Serradell MC, Torri A, Lujan HD. Putative SF2 helicases of the early-branching eukaryote Giardia lamblia are involved in antigenic variation and parasite differentiation into cysts. BMC Microbiol. 2012;12:284. doi:10.1186/1471-2180-12-284.

74. Fuller-Pace FV. DExD/H box RNA helicases: multifunctional proteins with important roles in transcriptional regulation. Nucleic Acids Res. 2006;34:4206-15. doi:10.1093/nar/gk1460.

75. Prucca CG, Slavin I, Quiroga R, Elías EV, Rivero FD, Saura A, et al. Antigenic variation in Giardia lamblia is regulated by RNA interference. Nature. 2008;456:750-4. doi:10.1038/nature07585.

76. Saraiya AA, Li W, Wang CC. A microRNA derived from an apparent canonical biogenesis pathway regulates variant surface protein gene expression in Giardia lamblia. RNA N Y N. 2011;17:215264. doi:10.1261/rna.028118.111.

77. Liao J-Y, Guo Y-H, Zheng L-L, Li Y, Xu W-L, Zhang Y-C, et al. Both endo-siRNAs and tRNA-derived small RNAs are involved in the differentiation of primitive eukaryote Giardia lamblia. Proc Natl Acad Sci U S A. 2014;111:14159-64. doi:10.1073/pnas. 1414394111. First study of changes in the level of siRNAs during encystation. tRNA fragments accumulate during the encystation process. 